\title{
Results of the LHCf experiment and the forward measurements at the LHC
}

T. Sako ${ }^{1,2, a}$, O. Adriani ${ }^{3,4}$, E. Berti ${ }^{3,4}$, L. Bonechi ${ }^{3}$, M. Bongi ${ }^{3,4}$, G. Castellini ${ }^{3,5}$, R. D’Alessandro ${ }^{3,4}$, M. Haguenauer $^{6}$, Y. Itow ${ }^{1,2}$, T. Iwata $^{7}$, K. Kasahara ${ }^{7}$, K. Masuda ${ }^{1}$, E. Matsubayashi ${ }^{1}$, H. Menjo ${ }^{8}$, Y. Muraki ${ }^{1}$, P. Papini ${ }^{3}$, S. Ricciarini ${ }^{3,5}$, N. Sakurai ${ }^{9}$, K. Sato ${ }^{1}$, M. Shinoda ${ }^{1}$, T. Suzuki ${ }^{7}$, T. Tamura ${ }^{10}$, A. Tiberio ${ }^{3,4}$, T. Torii ${ }^{7}$, A. Tricomi ${ }^{11,12}$, W.C. Turner ${ }^{13}$, M. Ueno ${ }^{1}$, and Q.D. Zhou ${ }^{1}$

${ }^{1}$ Institute for Space-Earth Environmental Research, Nagoya University, Nagoya, Japan

2 Kobayashi-Maskawa Institute for the Origin of Particles and the Universe, Nagoya University, Nagoya, Japan

3 INFN Section of Florence, Florence, Italy

${ }^{4}$ University of Florence, Florence, Italy

5 IFAC-CNR, Florence, Italy

${ }^{6}$ Ecole Polytechnique, Palaiseau, France

7 RISE, Waseda University, Shinjuku, Tokyo, Japan

8 Graduate School of Science, Nagoya University, Nagoya, Japan

9 Tokushima University, Tokushima, Japan

10 Kanagawa University, Kanagawa, Japan

11 INFN Section of Catania, Italy

12 University of Catania, Catania, Italy

13 LBNL, Berkeley, California, USA

\begin{abstract}
The Large Hadron Collider is intensively used to test hadronic interaction models used in cosmicray physics because its maximum (designed) collision energy $\sqrt{s}=14 \mathrm{TeV}$ corresponds to the interaction of a $10^{17} \mathrm{eV}$ cosmic-ray proton hitting the atmosphere. In this paper various types of forward experiments at the LHC, where the particles relevant to the air shower development are observed, are reviewed. Recent results of a dedicated experiment for precise forward measurement, the LHC forward, are discussed in detail. A future possibility at the LHC, light ion collisions, is also discussed.
\end{abstract}

\section{Introduction}

Interpretations of atmospheric air shower observations rely on Monte Carlo simulations, where we must assume a hadronic interaction model (see [1] for a review). However, because of the lack of knowledge both in theory and experiment of the high-energy hadronic interaction, there is an uncertainty in the modeling affects in the interpretations. The scope of this paper is to review the relation between the properties of hadronic interactions and air shower development, then focus on the measurements of forward particle production. Recent collider results, especially forward measurements at the LHC, are reviewed. The results of LHCf are discussed in detail.

\section{Air shower and accelerator experiments}

\subsection{Air shower development and hadronic interactions}

The properties of hadronic interactions can be categorized in a few steps from the cosmic-ray point of view.

a e-mail: sako@isee.nagoya-u.ac.jp
1. The total inelastic cross section, $\sigma_{\text {ine }}$, determines the mean free path of the interaction, and is directly related to the maximum depth of air shower, $\left\langle X_{\max }>\right.$.

2. After an inelastic interaction multi-particle production takes place, where the inelasticity determines the fractional energy carried by mesons, or elasticity, the fractional energy carried by the leading baryon.

3. Energy sharing between the mesons, energy spectra, or differential cross sections, are also important.

4. Nuclear effects to incorporate the atmosphere as the target and nuclear projectile.

5. Meson-air interactions are also important, but are not considered in this paper.

The effects of some properties, which can be parameterized, to the atmospheric air showers were studied in [2].

\subsection{Forward particle measurements at colliders}

Figure 1 shows the number and energy densities of the secondary particles in $\sqrt{s}=14 \mathrm{TeV} \mathrm{p}$-p inelastic interactions as functions of the pseudorapidity $\eta$. It is found that most of the particles are produced in the central rapidity, $|\eta| \sim 0$, but most of the energy flows in the forward, large $|\eta|$, directions. To understand the air shower development measurements of the energy flow,

(C) The Authors, published by EDP Sciences. This is an Open Access article distributed under the terms of the Creative Commons Attribution License 4.0 (http://creativecommons.org/licenses/by/4.0/). 

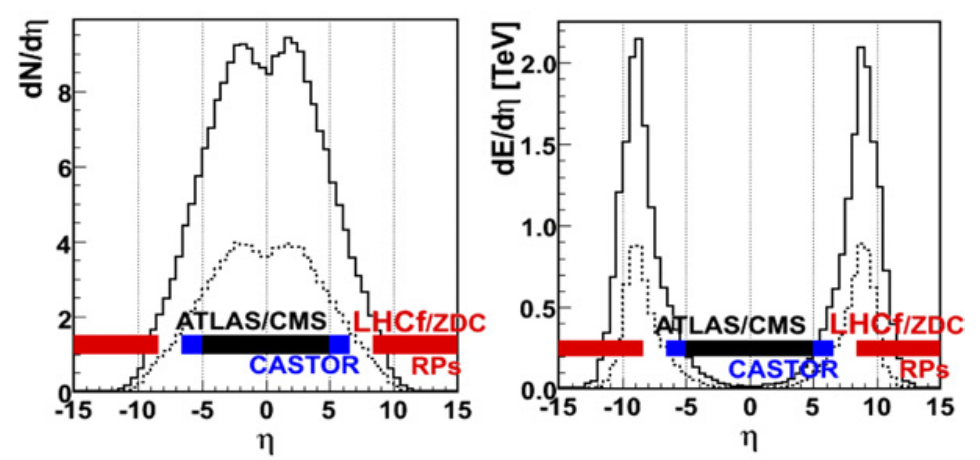

Figure 1. Number (left) and energy (right) densities of the secondary particles as functions of pseudorapidity $\eta$ in $\sqrt{s}=14 \mathrm{TeV}$ p-p collisions. Pseudorapidity coverages of some LHC experiments are shown by horizontal bands.

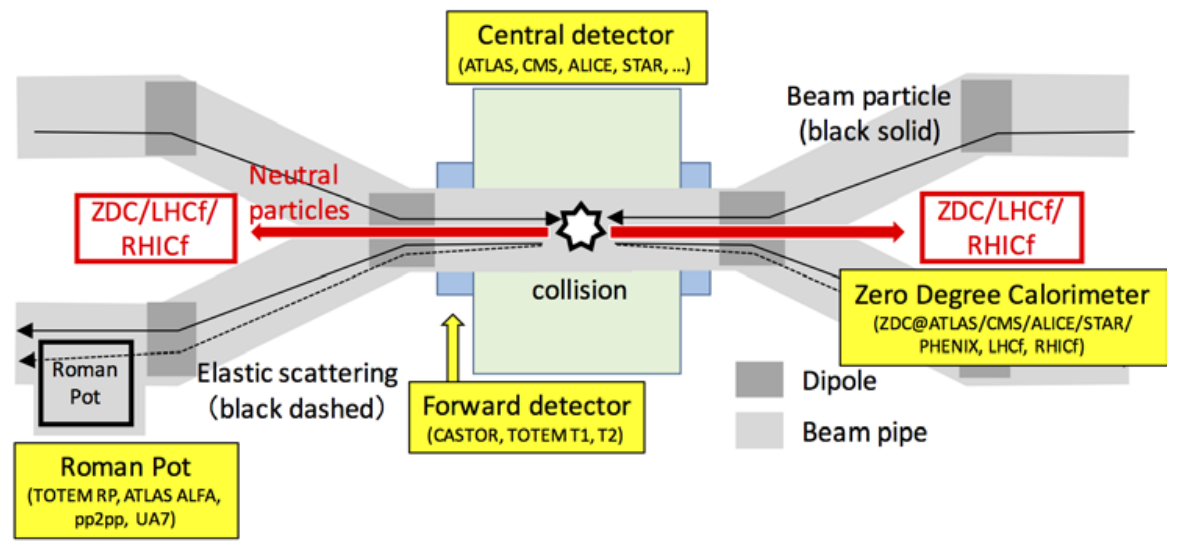

Figure 2. Schematic configuration of the forward detectors in the collider environment.

meaning the particle production in the forward direction, is essential. However, because forward directions are not covered by the main part of the general purpose detectors like ATLAS and CMS, dedicated experiments and detectors are required.

Forward experiments are classified in three cases according to their configurations as shown in Fig. 2.

1. As a part of the central detector but installed far from the interaction point and as close to the beam pipe as possible to detect inelastically scattered forward particles. CMS CASTOR [3] and TOTEM T1/T2 [4] at the LHC, for example, are categorized in this class.

2. Tracking detectors inserted into the vacuum beam pipes to measure the particles elastically scattered in very small angles. The insertion technique is called the Roman Pot, and TOTEM RP, ATLAS ALFA [5] at the LHC and pp2pp [6] at the RHIC are in this category. UA7 [7] at Spp̄S also used roman pots, but this experiment inserted calorimeters to measure inelastically produced photons.

3. Calorimeters installed between the beam pipes connected to the storage rings can measure neutral particles inelastically produced around zero degree. The detectors, called Zero Degree Calorimeters, were designed to measure the number of spectator neutrons in heavy ion collisions that are sensitive to the impact parameter of each collision. Similar types of ZDCs are installed in the ALICE [8], ATLAS [5] and CMS [3] experiments at the LHC, and PHENIX and STAR at the RHIC [9]. LHCf [10] and its extension RHICf [11] are a kind of ZDCs, but very different structure. LHCf was designed to precisely measure individual photons to identify $\pi^{0}$, but with a smaller aperture. Installation slots of ZDC are available only in particle-particle colliders, but not in the particle-antiparticle collider like the Tevatron.

\section{Forward measurements at LHC}

\subsection{Roman pot experiments}

One of the most important measurements at LHC for cosmic-ray physics is the determination of $\sigma_{\text {ine }}$. The central detectors measure $\sigma_{\text {ine }}$ by counting the number of interactions with as wide a coverage as possible, namely minimum bias events, but they still need a model-based extrapolation to estimate the number of interactions in which the produced particles escape from the detector acceptance [12-15]. The missing events are produced in the diffractive process and its theoretical prediction is difficult.

Instead of counting most of the inelastic interactions at the central rapidity, the roman pot experiments measure the elastic interactions at a very small scattering angle. By measuring the differential cross section $d \sigma / d t$ at small $t$, where $t$ is the momentum transfer of protons, the roman pot experiments can determine the total elastic cross section $\sigma_{\text {ela }}=\int(d \sigma / d t) d t$. Extrapolation of the differential cross section gives the value $\left.\frac{d \sigma}{d t}\right|_{t=0}$, and this is connected with the total cross section $\sigma_{t o t}$ through the optical theorem. Finally the total inelastic cross section is determined as $\sigma_{\text {ine }}=\sigma_{\text {tot }}-\sigma_{\text {ela }}$. 


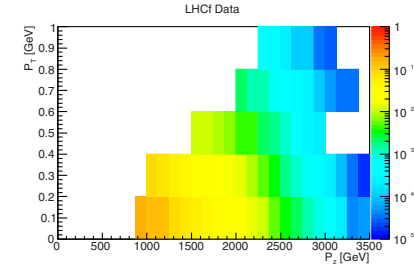

QGSJET II-04

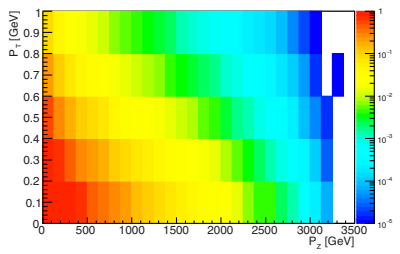

Figure 3. Invariant cross sections of $\pi^{0}$ production in the $\mathrm{p}_{z}-\mathrm{p}_{T}$ phase space in $7 \mathrm{TeV} \mathrm{p}-\mathrm{p}$ collisions. Invariant cross section $\left(1 / \sigma_{\text {ine }}\right) E d^{3} \sigma / d p^{3} \mathrm{GeV}^{-2}$ is shown in the color scale. LHCf result, predictions of EPOS-LHC, QGSJET II-04 and Sibyll 2.1 models are shown in the left-top, right-top, left-bottom and right-bottom panels, respectively.
Because $\sigma_{\text {ine }}$ had two different values in the Tevatron measurements [16-18], model predictions in the very highenergy region diverged significantly depending on the reference value used from the Tevatron results [19]. The improvement at the LHC measurements is not only that they are at the highest energy points, but also the results are consistent between experiments with small experimental uncertainties. Thanks to the convergence at the highest energy data, the post-LHC models like EPOS-LHC and QGSJET II-04 predict very similar $\sigma_{i n e}$ beyond the LHC energy, and the difference in the $\left\langle X_{\max }\right\rangle$ prediction is significantly reduced [19].

\subsection{The LHCf experiment}

The Large Hadron Collider forward (LHCf) experiment has two detectors called Arm1 and Arm2 at either side of IP1, $140 \mathrm{~m}$ away from the interaction point. Each detector is composed of two position sensitive electromagnetic calorimeters. Because dipole magnets are located between the LHCf detectors and the interaction point (Fig. 2), charged particles are swept away and only neutral particles, predominantly photons and neutrons, arrive at the detectors. The angular acceptance of the detectors is $\eta>8.4$. According to Fig. 1, it is found that the LHCf covers a large fraction of energy flow. The energy and position resolutions of the detectors are $<5 \%$ and $<200 \mu \mathrm{m}$, respectively, for photons with energy above $100 \mathrm{GeV}$ and $\sim 40 \%$ and $<1 \mathrm{~mm}$, respectively, for hadrons with energy above $500 \mathrm{GeV}$. By identifying the energies and positions of two photons, and assuming they decay at the interaction point, LHCf can identify $\pi^{0}$ with energies greater than $600 \mathrm{GeV}$.

LHCf so far obtained collision data for $\sqrt{s}=0.9,2.76$, 7 and $13 \mathrm{TeV}$ p-p collisions, $\sqrt{s_{N N}}=5.02$ and $8.16 \mathrm{TeV}$ $\mathrm{p}-\mathrm{Pb}$ collisions ${ }^{1}$. The forward photon spectra at 0.9 and $7 \mathrm{TeV}$ [20,21], forward neutron spectra at $7 \mathrm{TeV}$ [22], forward $\pi^{0}$ spectra at $7 \mathrm{TeV}$ [23] were already published. Forward $\pi^{0}$ spectra in the $5.02 \mathrm{TeV} \mathrm{p}-\mathrm{Pb}$ collisions were compared with the $\pi^{0}$ spectra interpolated from the 2.76 and $7 \mathrm{TeV}$ p-p collision data. Nuclear effects in terms of

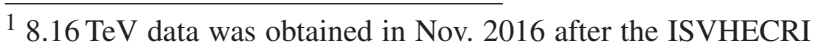
conference.
}

the nuclear modification factor were consistent with model predictions [24].

\section{$\pi^{0}$ cross section at $7 \mathrm{TeV}$}

Cross sections of $\pi^{0}$ production in the LHC Run1 data are summarized in [25]. A data-model comparison of $\pi^{0}$ production cross section in $\mathrm{p}_{z}-\mathrm{p}_{T}$ phase space in the $7 \mathrm{TeV}$ p-p collisions is shown in Fig. 3. The LHCf result in the left-top panel is compared with the predictions by EPOS-LHC (right-top), QGSJET II-04 (left-bottom) and Sibyll 2.1 (right-bottom) models. The ratio of the model predictions to the experimental result are shown in Fig. 4. A very flat ratio found in the QGSJET II-04 (left-bottom) means the spectral shape has very good agreement with the data, but the light blue to blue color means the cross section is underestimated over the whole kinematic region. EPOSLHC (right-top) shows good agreement below $2.5 \mathrm{TeV}$, above which energy the cross section is overestimated. Because the cross section at the high energy end is very small, the effect of this discrepancy to the air shower development will be small. Sibyll 2.1 shows a trend similar to EPOS-LHC. However, the overestimate is found in high $\mathrm{p}_{T}$ rather than high energy. As shown in Fig. 3, Sibyll 2.1 holds larger cross section at high $\mathrm{p}_{T}$ than the other models. Though the LHCf data constrains only at high energy, the flat $\mathrm{p}_{T}$ dependence of Sibyll is likely excluded by the LHCf data. The LHCf $13 \mathrm{TeV}$ data can extend the $\mathrm{p}_{T}$ coverage and can enforce this conclusion.

\section{Feynman scaling}

Cross sections of $\pi^{0}$ at $2.76 \mathrm{TeV}$ and $7 \mathrm{TeV}$ were compared in the same $\mathrm{x}_{F}$ and $\mathrm{p}_{T}$ region as shown in Fig. 5 [25]. Here the Feynman $\mathrm{x}, \mathrm{x}_{F}$, is defined as $2 p_{z} / \sqrt{s}$. The comparison shows a good agreement of the differential cross sections at the $20 \%$ level, indicating the existence of Feynman scaling [26]. On the other hand, when we compare the forward neutron cross sections between ISR at 30-60 GeV [27,28], PHENIX at $200 \mathrm{GeV}$ [29] and LHCf at $7 \mathrm{TeV}$ [22], the ISR and PHENIX results show a good scaling, but the LHCf result indicates a break of the scaling [30]. To test the Feynman scaling 

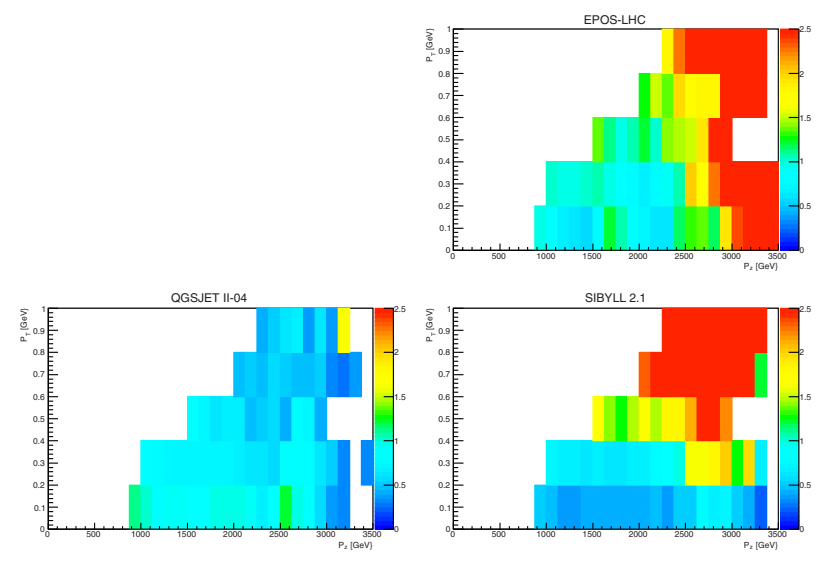

Figure 4. $\pi^{0}$ production cross sections by three models relative to the LHCf result. Ratio is shown in the color scale.

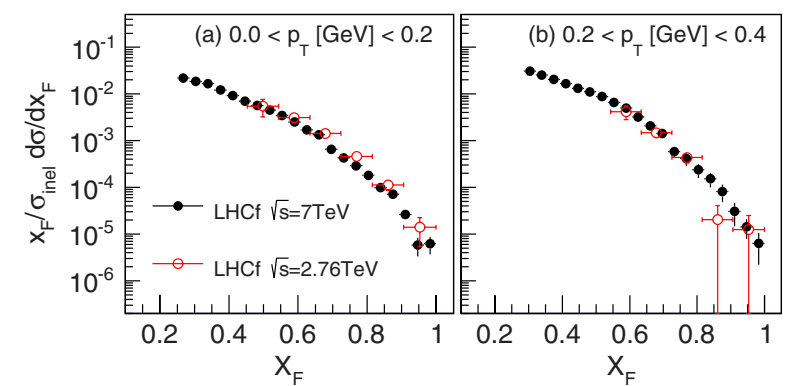

Figure 5. Cross sections of $\pi^{0}$ production measured at $2.76 \mathrm{TeV}$ and $7 \mathrm{TeV}$. Horizontal axis, $\mathrm{x}_{F}$, is normalized energy to the beam energy (see text). Data are compared in the same $\mathrm{p}_{T}$ ranges, a) $0.0<\mathrm{p}_{T}<0.2 \mathrm{GeV}$, b) $0.2<\mathrm{p}_{T}<0.4 \mathrm{GeV}$.

or its breaking, experiments at wider $\sqrt{s}$ coverage are important. Analysis of the LHCf $13 \mathrm{TeV}$ data and the RHICf experiment at $\sqrt{s}=510 \mathrm{GeV}$ [11] will extend such comparisons. Studies of $\sqrt{s}$ dependence are important to interpolate and extrapolate the accelerator data to the energy ranges of the knee and the GZK, respectively.

\section{Prospect for ATLAS-LHCf joint analysis}

The origin of forward particle production is roughly classified in two cases; diffraction and non-diffraction. Contribution from two different origins in the LHCf photon observations are studied by using different interaction models as shown in Fig. 6 [31]. It is interesting that the two processes contribute to the LHCf result almost equally and the detail is model dependent. Because the fundamental physics is very different between the two processes, experimental classification of the LHCf data provides a new clue to improve the models. In [31], a simple method to classify the LHCf data using the information of the ATLAS tracker is proposed. ATLAS and LHCf have successfully taken common data in the $13 \mathrm{TeV}$ operation, and new analysis of forward-central correlation is in discussion.

\subsection{Other forward measurements}

The CMS and TOTEM experiments report the particle number density and energy density in a wide pseudorapi-

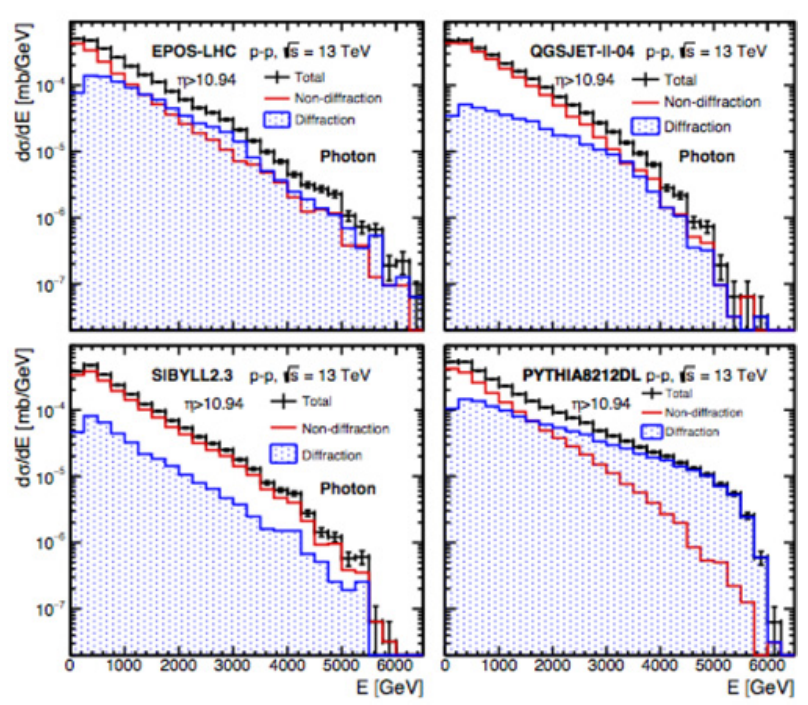

Figure 6. Cross sections of forward photon production in $13 \mathrm{TeV}$ collisions predicted by EPOS-LHC (left-top), QGSJET II-04 (right-top), Sibyll 2.3 (left-bottom) and PYTHIA 8212DL (rightbottom). Black histograms are inclusive cross sections while blue and red show the contributions from diffractive and nondiffractive processes, respectively.

tity range. The number density in the interval $0<\eta<7$ at $\sqrt{s}=8 \mathrm{TeV}$ shows generally good agreement with the model predictions [32]. Energy density distributions at $\sqrt{s}=7 \mathrm{TeV}$ and $13 \mathrm{TeV}$ are reported in [33] and [34], respectively. Although the predictions by the cosmic-ray models show good agreements with the $7 \mathrm{TeV}$ data, a systematic difference is found in the $13 \mathrm{TeV}$ results.

The ATLAS experiment reports a comparison between the $13 \mathrm{TeV}$ data and model predictions for the number of hits in the minimum bias trigger scintillators (MBTSS) [15]. The MBTSs cover the beam pipe in the forward direction with an azimuthal segmentation. Differently from the other minimum bias data, the predictions by EPOS-LHC and QGSJET II-04 have a significant discrepancy from the data. Because the number of hits is related to the correlation in the multi-particle production, this data will give a new view point to test the hadronic interaction models. 

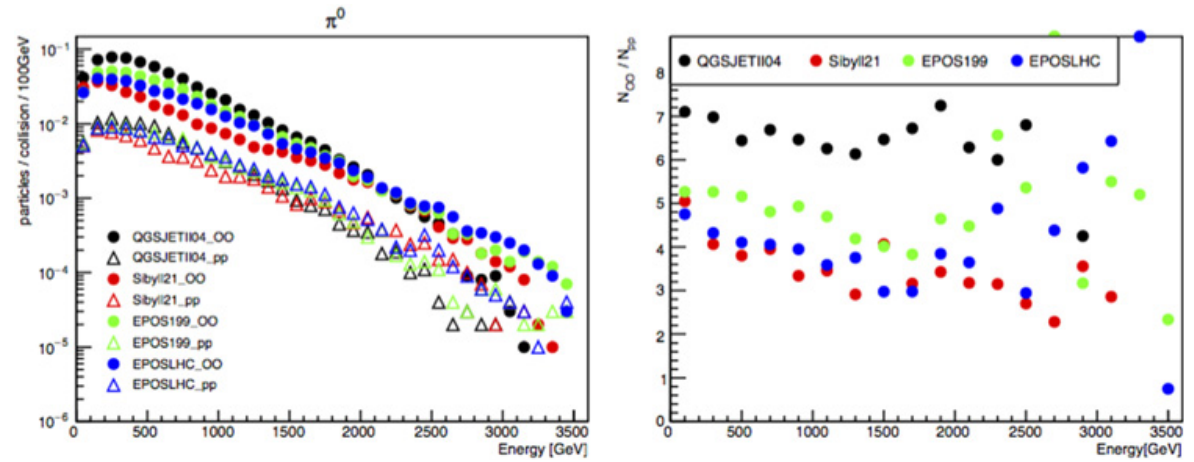

Figure 7. Left: forward $\pi^{0}$ production rates in the $\sqrt{s_{N N}}=7 \mathrm{TeV}$ p-p collisions (open triangles) and O-O collisions (filled circles) at $\eta>8.78$. Right: ratio of the production rates in the O-O collisions divided by the rates in the p-p collisions. Black, red, green and blue markers show the results of QGSJET II-04, Sibyll 2.1, EPOS 1.99 and EPOS-LHC interaction models, respectively.

\section{Possibility of Oxygen collisions at LHC}

The ultimate goal to use the LHC for cosmic-ray physics is to collide light ions with various kinds of ions, ranging from protons to Iron. A possibility of light ion operation at the CERN accelerator complex was studied and presented in [35]. They concluded the acceleration and collisions of the Oxygen beam is technically feasible when low luminosity collisions are accepted. A study of the existing model uncertainty in the $\mathrm{p}-\mathrm{O}$ collisions was given in [19]. Although the post-LHC models, EPOS-LHC and QGSJET II-04, have good agreement for the p-p collisions in $d N / d \eta$, they have clear differences in predictions of $\mathrm{p}-\mathrm{O}$ collisions.

Light ions represent not only the atmospheric target, but also cosmic-ray primary particles. A study of the O-O collisions was also made by the LHCf group. Figure 7 (left) shows the model predictions of the forward $\pi^{0}$ production rates in $\sqrt{s_{N N}}=7 \mathrm{TeV}$ p-p collisions and O-O collisions at $\eta>8.78$ The $\mathrm{p}-\mathrm{p}$ collisions are calculated to extract the model dependence of nuclear effects by dividing the $\mathrm{O}-\mathrm{O}$ results with the $p-p$ results. The ratios are shown in the right panel of Fig. 7. It is found that the EPOS-LHC and QGSJET II-04 show a factor 1.5-2 difference in the ratio, or in the nuclear effect.

\section{Summary}

The LHC experiments are extensively studying minimum bias events with various types of forward detectors. Some interaction models have been improved using the LHC Run1 data and they improved the interpretation of the cosmic-ray data. New data from $13 \mathrm{TeV}$ collisions become available from the LHC. Not only the multiplicity distribution, but also various new comparisons with data to enable further improve the models. Comparisons of data in a wide $\sqrt{s}$ range are also important to access energies beyond the LHC. Discussions for future light ion collisions must be continued.

\section{References}

[1] K.-H. Kampert and M. Unger, Astropart. Phys. 35 (2012) 660-678

[2] R. Ulrich, R. Engel, M. Unger, Phys. Rev. D 83 (2011) 054026
[3] The CMS Collaboration, JINST 3 (2008) S08004

[4] The TOTEM Collaboration, JINST 3 (2008) S08007

[5] The ATLAS Collaboration, JINST 3 (2008) S08003

[6] S. Bültmann et al., Nucl. Instrum. Methods A 535 (2004) 415-420

[7] The UA7 Collaboration, Phys. Lett. B 242 (1990) 531-535

[8] The ALICE Collaboration, JINST 3 (2008) S08002

[9] C. Adler et al., Nucl. Instrum. Methods A 499 (2002) 433-436

[10] The LHCf Collaboration, JINST 3 (2008) S08006

[11] The RHICf Collaboration, arXiv: 1409.4860

[12] The ATLAS Collaboration, Nature Commun. 2 (2011) 463

[13] The CMS Collaboration, Phys. Lett. B 722 (2013) 5

[14] The ALICE Collaboration, Eur. Phys. J. C 73(6) (2013) 2456

[15] The ATLAS Collaboration, ATLAS-CONF-2015038

[16] The E710 Collaboration, Phys. Rev. Lett. 68 (1992) 2433-2436

[17] The CDF Collaboration, Phys. Rev. D 50 (1994) 5550-5561

[18] The E811 Collaboration, Phys. Lett. B 445 (1999) 419-422

[19] K. Akiba et al., J. Phys. G: Nucl. Part. Phys. 43 (2016) 110201

[20] The LHCf Collaboration, Phys. Lett. B 715 (2012) 298-303

[21] The LHCf Collaboration, Phys. Lett. B 703 (2011) 128-134

[22] The LHCf Collaboration, Phys. Lett. B 750 (2015) 360-366

[23] The LHCf Collaboration, Phys. Rev. D 86, 092001 (2012)

[24] The LHCf Collaboration, Phys. Rev. C 89, 065209 (2014)

[25] The LHCf Collaboration, Phys. Rev. D 94, 032007 (2016)

[26] R. P. Feynman, Phys. Rev. Lett. 23, 1415 (1969)

[27] J. Engler, et al., Nucl. Phys. B 84 (1975) 70

[28] W. Flauger, F. Mönnig, Nucl. Phys. B 109 (1976) 347-356

[29] The PHENIX Collaboration, Phys. Rev. D 88 (2013) 032006 
[30] K. Kawade, PhD thesis, Nagoya University (2014); CERN-THESIS-2014-315

[31] Q.D.Zhou, Y. Itow, H. Menjo and T. Sako, arXiv: 1611.07483v2 [hep-ex]

[32] The TOTEM Collboration, EPJC (2015) 75, 126

[33] The CMS Collboration, JHEP 11 (2011) 148
[34] The CMS Collboration, CMS-PAS-FSQ-15-006

[35] D. Manglunki, presented in the "Results and prospects of forward physics at the LHC; Implications for the study of diffraction cosmic ray interactions, and more," https://indico.cern. ch/event/223562/contributions/470231/ 